\title{
Lösungsmöglichkeiten bei fehlgeschlagenen Osteosynthesen - Ellenbogen
}

\author{
Frank Graef, Ulrich Stöckle, Kathi Thiele
}

\section{Einleitung}

Der Ellenbogen ist sowohl anatomisch als auch biomechanisch-funktionell ein wichtiger und komplexer Teil der Bewegungskette der oberen Extremität. Anatomisch liegen hier neurovaskuläre, muskulotendinöse und ossäre Strukturen eng zusammen [1]. Das Gelenk besteht aus 3 Kompartimenten und verbindet den distalen Anteil des Humerus mit dem proximalen Anteil von Ulna und Radius. Das Humeroulnargelenk ist ein Scharniergelenk und ermöglicht Extension und Flexion. Das Humeroradialgelenk ist ein Eigelenk und lässt sowohl Extension und Flexion sowie Pro- und Supination zu. Diese Umwendbewegung wird letztlich auch durch die Artikulation von Ulna und Radius im Ulnoradialgelenk ermöglicht, einem Radgelenk.

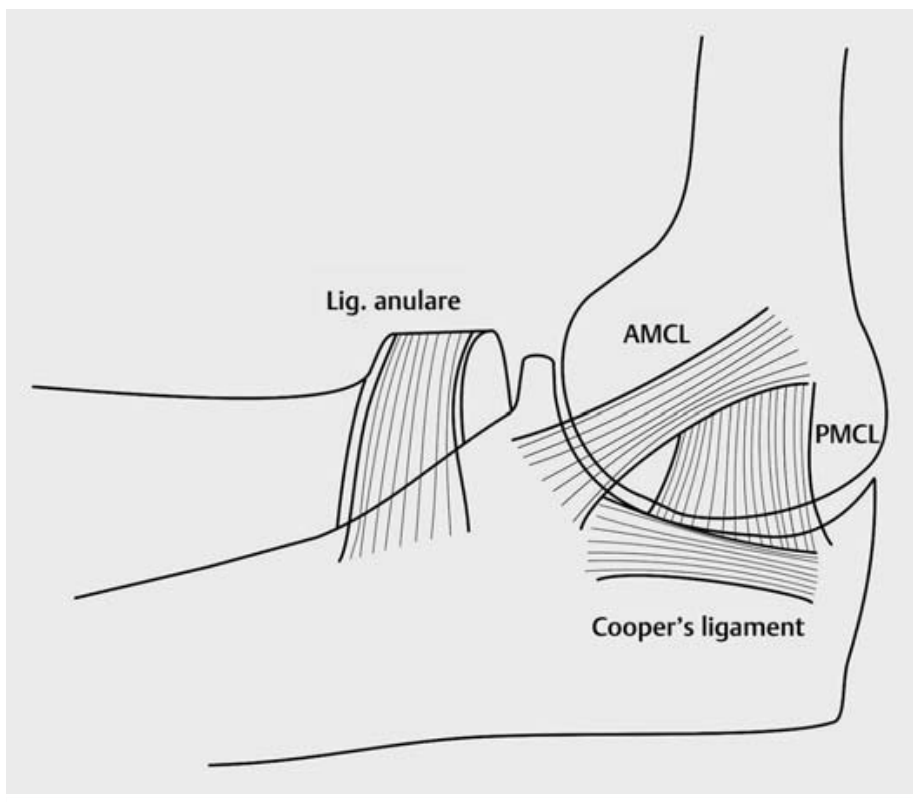

- Abb. 1 Ellenbogengelenk seitlich von ulnar betrachtet. Das mediale kollaterale Ligament (MCL) besteht aus dem anterioren Bündel (AMCL), dem posterioren Bündel (PMCL) sowie dem transversalen Bündel („Cooper's ligament“). Dabei ist das AMCL das funktionell wichtigste Ligament und primärer Stabilisator des Ellenbogengelenks gegen Valgusstress. Das Ligament setzt an der anteromedialen Facette des Coronoids an. Coronoidfrakturen, welche die anteromediale Facette betreffen, sind dementsprechend stabilitätsgefährdet.
Die Stabilität des Gelenks wird auf der einen Seite durch eine hohe Kongruenz der knöchernen Gelenkpartner und auf der anderen Seite durch ein komplexes KapselLigament-System gewährleistet. Zu den primären Stabilisatoren des Ellenbogengelenks zählen:

- das Humeroulnargelenk,

- der laterale Kollateralbandkomplex (LCL) und

- der mediale ulnare Kollateralbandkomplex (MUCL).

Sekundäre Stabilisatoren sind:

- das Humeroradialgelenk,

- die Extensorenmuskulatur (Ursprung am Epicondylus humeri lateralis),

- die Flexorenmuskulatur (Ursprung am Epicondylus humeri medialis) und

- die Gelenkkapsel [2].

Das mediale Kollateralband (MCL) besteht aus dem anteromedialen Kollateralband (AMCL), dem posteromedialen Bündel (PMCL) und dem transversalen Bündel („Cooper's ligament“). Eine besonders wichtige Funktion kommt hierbei dem AMCL zu, es ist der primäre Stabilisator des Ellenbogens gegen Valgusstress zwischen 20 und $120^{\circ}$ Flexion [3]. Es hat seinen Ursprung an der anteriorinferioren Facette des Epicondylus medialis und setzt an der anteromedialen Facette (Tuberculum subliminus) des Processus coronoideus an ( $\bullet$ Abb. 1 ). Entsprechend ist die anteromediale Facette des Coronoids wichtig hinsichtlich der Stabilität des Gelenks bei Frakturen in diesem Bereich. Werden diese nicht suffizient behandelt, kann als Komplikation eine posteromediale Rotationsinstabilität auftreten [4]. Diese anatomische Besonderheit ist auch Grundlage der Klassifikation von O’Driscoll für Coronoidfrakturen [4].

Der laterale Kollateralbandkomplex (LCL) besteht aus dem radialen Seitenband ( $R C L)$, dem lateralen ulnaren Seitenband (LUCL) und dem Lig. anulare. Der LCL entspringt am Unterrand des Epicondylus lateralis aus einem gemeinsamen Isometriepunkt [5]. Dieser gemeinsame Isometriepunkt projiziert sich auf das Zentrum des Capitulums, nicht des lateralen Epicondylus. Hier ist bei Rekonstruktionen das Band auch zu refixieren. Das LUCL ist der wichtigste Stabilisator gegen Varusstress und setzt an der Crista supinatoris der Ulna an. Dabei verläuft es dorsal des Radiuskopfes, dadurch wird es umschlossen und stabilisiert [2, 6] ( A Abb. 2). 
Bei der Rekonstruktion von Frakturen der proximalen Ulna ist auf eine akkurate Wiederherstellung der physiologischen Anatomie zu achten. Dabei spielt die dorsale Angulation der proximalen Ulna eine große Rolle. Radiologisch gemessen wird diese anhand des PUDA-Winkels (PUDA = proximal ulna dorsal angulation). Der Winkel wird gemessen, indem am seitlichen Röntgenbild des Ellenbogens eine Tangente an den dorsalen Aspekt des Olekranons und eine weitere Tangente an die dorsale Kante der Ulna gelegt wird ( $\triangleright$ Abb. 3). Der PUDA beträgt physiologisch im Mittel 5,7 ${ }^{\circ}$ [7]. Wird der PUDA nicht korrekt rekonstruiert, kann es zu Streckdefiziten sowie einer insuffizienten Kongruenz des radioulnaren Gelenks mit entsprechender Malrotation des Unterarms kommen [7]. Insbesondere bei Monteggia-Verletzungen ist der PUDAWinkel zu beachten, da eine insuffiziente Rekonstruktion zu einer chronischen Instabilität im Humeroradialgelenk führen kann (siehe unten, Monteggia-Verletzungen) [8]. Weitere wichtige Winkel des Ellenbogengelenks sind einerseits die ventrale Angulation des Capitulums von $30^{\circ}$ sowie der Cubitus-Valgus-Winkel (engl. carrying angle), der die physiologische valgische Achsabweichung des Unterarms im a.-p. Röntgen beschreibt und in der Literatur mit Werten zwischen 10 und $20^{\circ}$ angegeben wird ( $\triangleright$ Abb.3). [9]. Eine weitere wichtige Orientierung für die spätere Rekonstruktion ist die Beziehung zwischen der Spitze des Processus coronoideus und des Olekranons, wobei eine gezeichnete Linie zwischen diesen in einem $30^{\circ}$-Winkel zur Ulnaachse im seitlichen Bild steht. Bei Patienten mit unilateraler Verletzung ist aufgrund der hohen Varianz dieser Winkelangaben vor operativer Versorgung ein Röntgenbild der Gegenseite zum Vergleich durchzuführen.

\section{Pathologien}

\section{Proximale Radiusfrakturen}

Zu den proximalen Radiusfrakturen zählen die Radiuskopffraktur, die Radiushalsfraktur sowie entsprechende ligamentäre Verletzungen. Die Verletzungen treten meist beim Sturz auf den ausgestreckten Arm auf, dabei spielen typischerweise 2 Unfallmechanismen eine Rolle:

1. Sturz mit Valgusstress führt zu axialem Druck des Radiuskopfes auf das Capitulum und dementsprechend zu Radiuskopfimpaktionsfrakturen, häufig mit MCLRupturen vergesellschaftet.

2. Posterolaterale Subluxation des Radiuskopfes führt zu Scherfrakturen des Radiuskopfes am Capitulum [10].

Dementsprechend ist bei der primären Versorgung des Patienten auf ligamentäre Instabilitäten zu achten. Anatomisch ist der Radiuskopf artikulär komplett und zu $280^{\circ}$ zirkumferent von Gelenkknorpel überzogen, da er mit Humerus und Ulna artikuliert. Die meisten Frakturen sind im Bereich der lateralen bzw. anterolateralen Region des Radiuskopfes bei fehlender chondraler Deckung zu

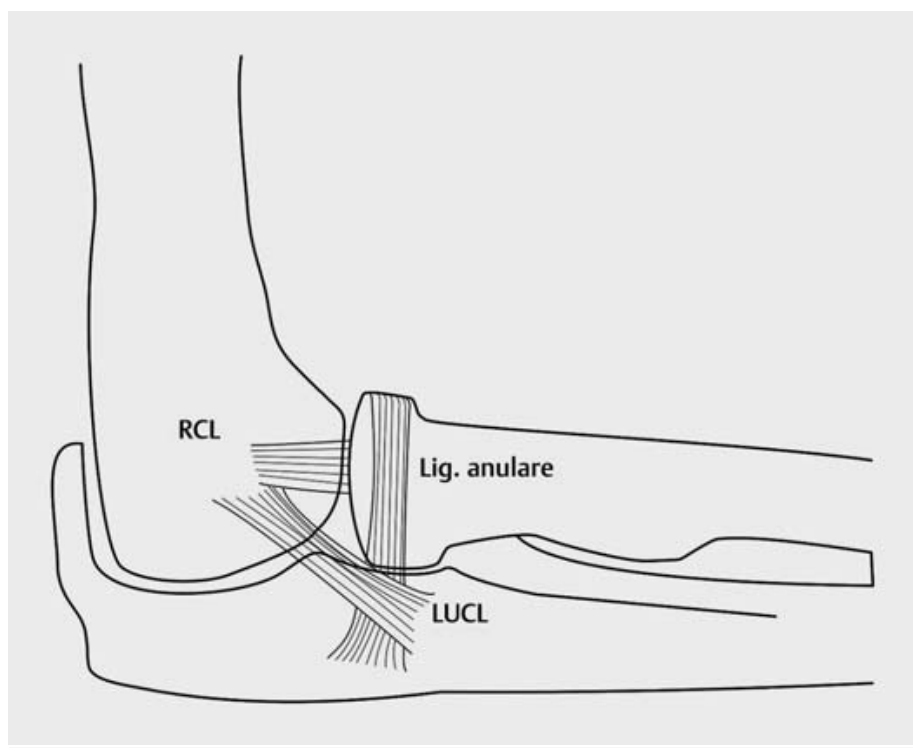

- Abb. 2 Ellenbogengelenk seitlich von lateral betrachtet. Die dorsale Begrenzung des Ellenbogens wird durch das Olekranon gebildet, auf der Ulna reitet im proximalen radioulnaren Gelenk der Radiuskopf. Das laterale kollaterale Ligament $(\mathrm{LCL}$ ) besteht aus dem radialen kollateralen Band $(R C L)$, dem Lig. anulare und dem lateralen ulnaren kollateralen Band (LUCL). Letzteres ist für die Primärstabilität bei Varusstress verantwortlich.
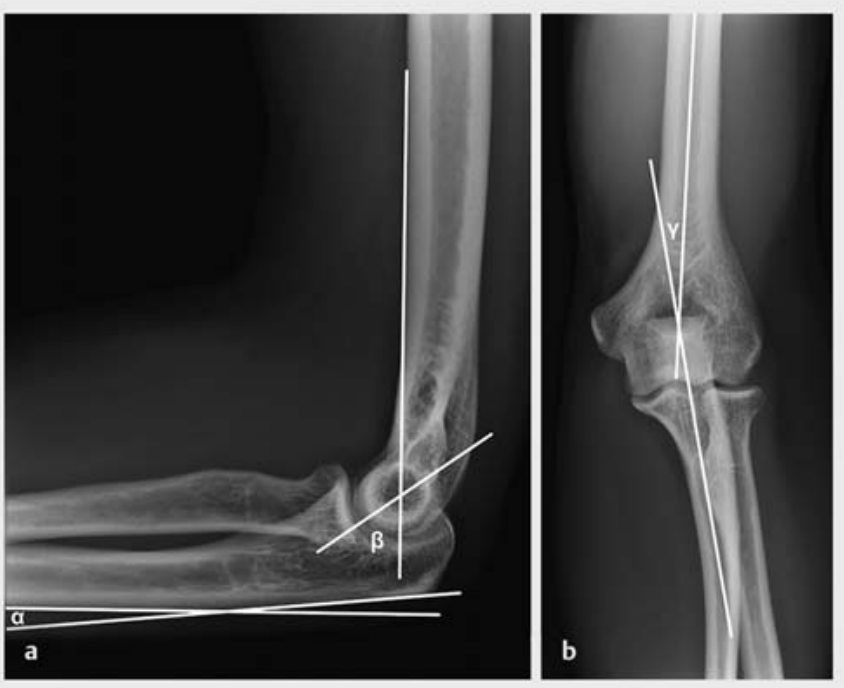

- Abb. 3 Ellenbogengelenk seitlich und a.-p. Im seitlichen Röntgenbild ist zum einen mit $\alpha$ der PUDA-Winkel (PUDA = proximal ulna dorsal angulation) und andererseits mit $\beta$ die anteriore Angulation des Capitulums eingezeichnet. Im a.-p. Röntgenbild ist der Cubitus-Valgus-Winkel (engl. carrying angle) mit y eingezeichnet. Die Rekonstruktion dieser Landmarks ist insbesondere im Revisionsfall wichtig, bei unilateraler Verletzung sollten vergleichende Röntgenbilder der Gegenseite angefertigt werden. 
finden. Um insbesondere bei Plattenosteosynthesen kein implantatbedingtes Impingement zwischen Ulna und Radius zu provozieren, ist auf die sog. „Safe Zone“ zu achten, die sich in Neutralstellung nach lateral darstellt [11]. Radiuskopffrakturen werden nach Mason unterteilt. TypI-Verletzungen stellen nicht dislozierte Frakturen mit einem Frakturspalt $<2 \mathrm{~mm}$ dar, bei Typ-II-Frakturen ist der Frakturspalt > 2 mm disloziert und partiell intraartikulär, Typ-III-Frakturen sind vollständig intraartikuläre Frakturen [12].

Bei Patienten mit Osteosyntheseversagen am proximalen Radius ist zunächst ein vollständiges Work-up des Patienten obligat. Dies schließt eine klinische Untersuchung des Ellenbogens mit Überprüfung der ligamentären Stabilität, eine Blutentnahme, Röntgen- sowie CT-Aufnahmen ein. Wie alle Frakturen im Bereich des Ellenbogens gestalten sich Revisionseingriffe aufgrund der hohen Kongruenz der Gelenkpartner und des komplexen Bandapparates schwierig. Deshalb ist eine korrekte Versorgung schon beim Ersteingriff wichtig. Im Bereich des Radiuskopfes ist hierbei auf eine exakte anatomische Reposition und suffiziente Osteosynthese zu achten. Partiell intraartikuläre und einfache Frakturen können durch isolierte Schraubenosteosynthesen stabilisiert werden. Komplexe, vollständig intraartikuläre Frakturen sollten, aufgrund des ebenfalls frakturierten Radiushalses, mittels offener Reposition und einer winkelstabilen Plattenosteosynthese versorgt werden. Radiushalsfrakturen sind entsprechend den AO-Prinzipien (AO: Arbeitsgemeinschaft für Osteosynthesefragen) durch eine Plattenosteosynthese zu operieren [13]. Liegt ein Osteosyntheseversagen aufgrund einer insuffizienten Reposition und Fixation vor, ist bei vitalem Knochen eine Reosteosynthese mit Anstrebung einer suffizienten Stabilität durch winkelstabile Platten möglich [10]. Bei Pseudarthrosen mit vergesellschafteter Radiuskopfnekrose ist eine Reosteosynthese häufig nicht mehr möglich. Dann empfiehlt sich die Implantation einer Radiuskopfprothese. Hierbei ist die Examinierung und ggf. Versorgung des Bandapparates obligat für eine erfolgreiche Revision ( $\bullet$ Abb. 4).

\section{Proximale Ulna}

Zu den proximalen Ulnafrakturen werden die Olekranonfraktur, die Monteggia- bzw. Monteggia-like-Frakturen sowie Frakturen des Processus coronoideus gezählt. Letztere werden in diesem Artikel unter dem Punkt Ellenbogenluxationsfrakturen besprochen. Olekranonfrakturen entstehen i.d. R. durch ein direktes Trauma mit Sturz auf den $90^{\circ}$ gebeugten Ellenbogen und stellen mit ca. $10 \%$ aller Frakturen an der oberen Extremität eine häufige Verletzung dar $[10,14]$. Für Olekranonfrakturen existieren mehrere Klassifikationen wie die Schatzker-Klassifikation, die AO-Klassifikation und die Mayo-Klassifikation [15]. Prinzipiell muss bei der Wahl der Osteosynthese zwischen stabilen und instabilen Frakturen unterschieden werden. Als stabil können transversal verlaufende 2-Part-Fraktu- ren gesehen werden und/oder Frakturen, die auf Höhe bzw. proximal des Mittelpunktes der Incisura trochlearis lokalisiert sind. Frakturen mit Trümmerzone, Subluxationen/Luxationen oder Frakturen, die distal des Mittelpunktes der Incisura trochlearis liegen, sind instabil [15]. Stabile Frakturen können prinzipiell mittels Zuggurtungsosteosynthese versorgt werden. Dabei muss jedoch die Indikation stimmen und die Operation technisch korrekt ausgeführt werden. Der häufigste Grund für Implantatversagen bei Zuggurtungsosteosynthesen ist auf eine falsche Durchführung bzw. falsche Indikation für dieses Osteosyntheseverfahren zurückzuführen [14]. Eine häufige Komplikation besteht in der insuffizienten Refixation weit proximaler Avulsionsfrakturen, die durch die Osteosynthese nicht adäquat adressiert werden und im weiteren Verlauf dislozieren. Hier empfiehlt sich die Verwendung von anatomisch vorgeformten Plattensystemen bis hin zu Doppelplattenosteosynthesen. Bei instabilen Frakturen oder im Revisionsfall sollte in jedem Fall eine plattenosteosynthetische Versorgung erfolgen. Da es sich um Gelenkfrakturen handelt, ist die anatomische Rekonstruktion mit interfragmentärer Kompression sowie absoluter Stabilität obligat. Auch hierbei sind proximal gelegene Fragmente mit zu erfassen, da diese sekundär durch den Zug des M. triceps brachii dislozieren können ( Abb. 5).

Bei Monteggia-Frakturen handelt es sich klassischerweise um die Kombination aus einer proximalen Ulnafraktur und der anterioren Luxation des Radiuskopfes. Bado erweiterte den Begriff der Monteggia-Frakturen um 4 Phänotypen [16]: Typ I: proximale Olekranonfraktur mit anteriorer Luxation des Radiuskopfes, Typ II mit posteriorer Luxation des Radiuskopfes, Typ III mit lateraler Luxation des Radiuskopfes und Typ IV mit begleitender Radiusschaftfraktur. Die Jupiter-Subklassifikation erweitert die Bado-Klassifikation von Typ II [17]: Typ IIA: Die Ulnafraktur betrifft das Olekranon und das Coronoid. Typ IIB: Die Ulnafraktur befindet sich distal des Coronoids im Bereich des Übergangs Metaphyse-Diaphyse. Typ IIC: Die Ulnafraktur ist im Bereich der Diaphyse. Typ IID: Die Fraktur betrifft den gesamten Bereich des proximalen Drittels der Ulna [8]. Bei der offenen Reposition und Fixation der Ulnafraktur ist bei Monteggia-Frakturen auf die exakte anatomische Reposition zu achten. Bei insuffizienter Reposition und Fixation kann eine chronische Radiuskopfluxation resultieren [18]. Intraoperativ ist deshalb zu prüfen, ob der Radiuskopf suffizient reponiert wird und stabil im Gelenk verbleibt, das Ellenbogengelenk ist entsprechend nach der osteosynthetischen Versorgung auf eventuelle Instabilitäten hin zu überprüfen. Ist eine suffiziente Reposition des Radiuskopfes trotz korrekter Reposition der Ulna nicht möglich, so kann in manchen Fällen das Lig. anulare als Repositionshindernis stören. Das Ligament muss in diesem Fall zur Reposition eröffnet und anschließend wieder vernäht werden. Bei der Rekonstruktion von Monteggia-Frakturen, insbesondere im Revi- 


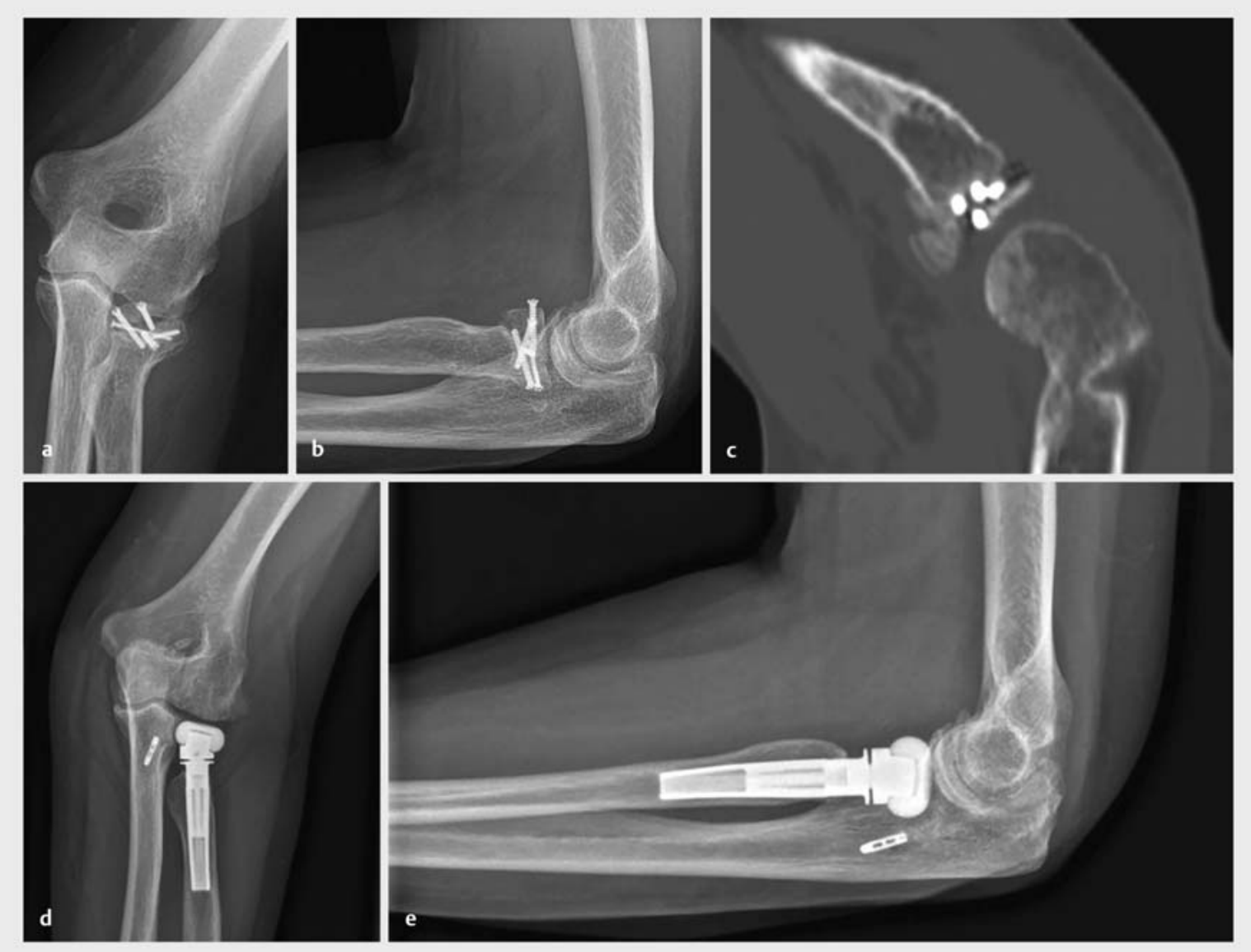

- Abb. 4 Zustand nach auswärtiger Schraubenosteosynthese einer vollständig intraartikulären Mason-3-Fraktur einer 53-jährigen Patientin. Im Verlauf zeigte sich nach 8 Monaten eine Pseudarthrose mit Osteosyntheseversagen, intraartikulärer Schraubenlage und Nekrose des Radiuskopfes (a-c). Zudem lag eine traumatische und mittlerweile chronische Insuffizienz des lateralen ulnaren Bandapparates vor. Durch eine Staging-Arthroskopie konnte eine Infektion ausgeschlossen werden. 10 Monate nach Trauma erfolgte der Revisionseingriff mit Implantation einer Radiuskopfprothese (Typ Mopyc, Fa. Tornier) sowie Rekonstruktion des LUCL mit Fascia lata in modifizierter Jobe-Technik (ulnar Endobutton, humeral Sviwelock 4.75) (d, e).

sionsfall, ist auf die korrekte Reposition der Ulnafraktur zu achten. Bei Bado-Typ-I-Verletzungen kommt es korrespondierend zur anterioren Luxation des Radiuskopfes zu einer dorsalen Angulation der Ulna. Wird der PUDA-Winkel nicht korrekt rekonstruiert ( $\bullet$ Abb. 3), kann sich eine chronische Instabilität im Humeroradialgelenk entwickeln. Bei Bado-Typ-II-Verletzungen kommt es entsprechend der posterioren Radiuskopfluxation zu einer ventralen Angulation der Ulna, die entsprechend korrigiert werden muss [8]. Neben der Achskorrektur im seitlichen Alignment, ist weiterhin eine radiale Plattenanlage zu vermeiden. Dies führt neben der Verstärkung des BowingEffektes auch zu einer Einschränkung der Umwendbewegung. Bei Osteosyntheseversagen bzw. fehlender knöcherner Konsolidierung ist die Lösung häufig die Revision mittels Reosteosynthese unter Verwendung von Autobzw. Allografts.

\section{Distaler Humerus}

Die distale Humerusfraktur stellt eine seltene Verletzungsentität dar, die in ihrer Versorgung häufig extrem anspruchsvoll ist. Sie macht ca. 2-6\% aller Frakturen bei einer Inzidenz von 5,7 pro 100000 Einwohnern pro Jahr aus [19]. Die anatomische Komplexität, die limitierte knöcherne Substanz des distalen Humerus wie auch die anatomische Nähe der neurovaskulären Strukturen erschweren zusätzlich die eigentliche Frakturversorgung. Grundlage einer erfolgreichen Versorgung ist die stabile anatomische Rekonstruktion unter Beachtung der 3-dimensionalen Achsenstruktur des distalen Humerus. Die Anatomie des distalen Humerus entspricht einer Triangel, wobei die Seiten durch den medialen und lateralen Pfeiler definiert sind und die horizontale Achse dem artikulären Anteil entspricht. Bei der Rekonstruktion ist zu beachten, dass die artikuläre Fläche einer Innenrotation von ca. 5$7^{\circ}$, einem Valgus von $5-8^{\circ}$ und einer Anteflexion von $30^{\circ}$ 

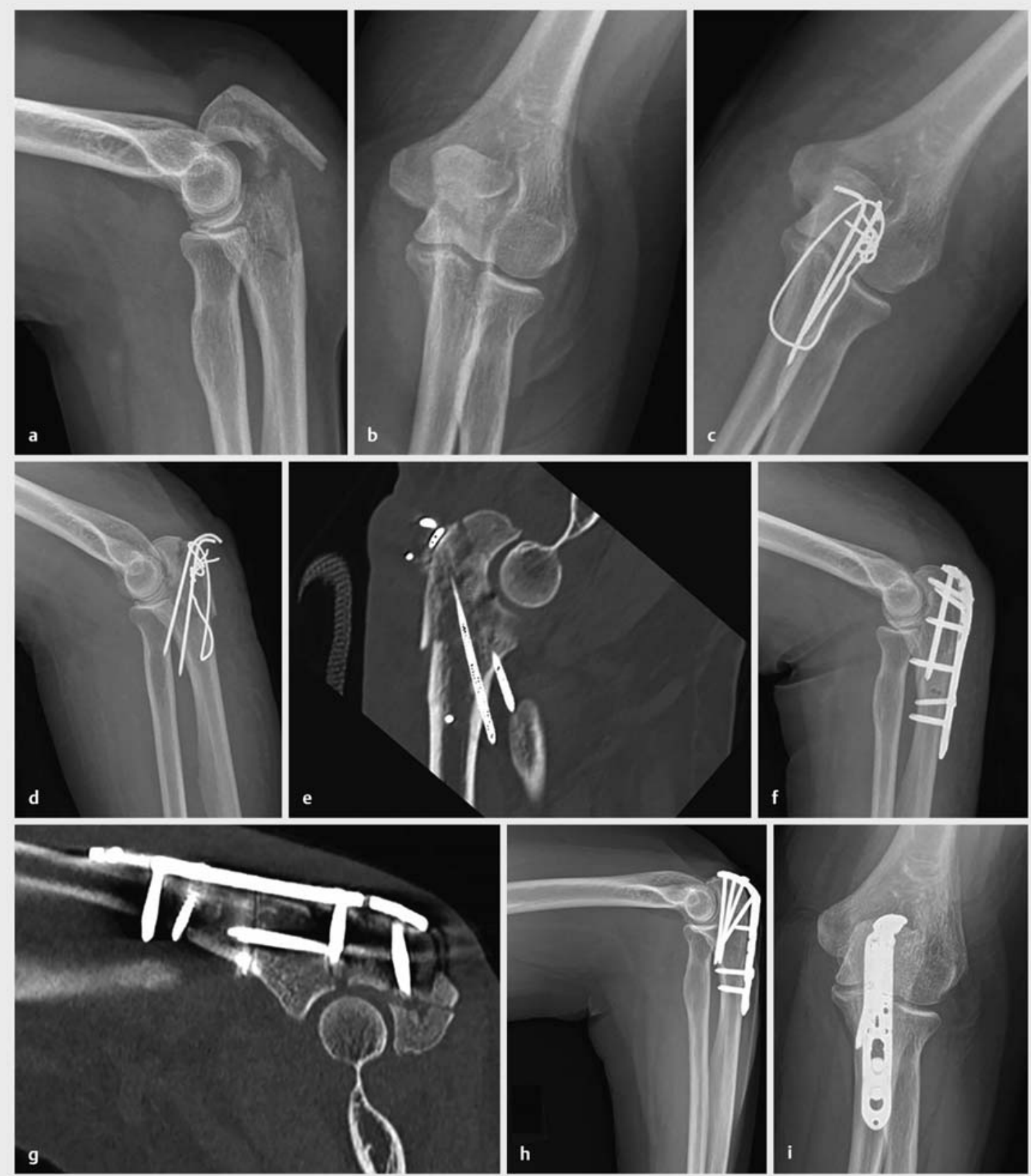

- Abb. 5 Fall einer 70-jährigen Patientin, die initial in einer auswärtigen Klinik bei instabiler und multifragmentärer Olekranonfraktur mittels Zuggurtungsosteosynthese versorgt wurde. Im postoperativen Röntgen sowie CT zeigte sich eine insuffiziente Versorgung mit sekundärer Dislokation, sodass in derselben Klinik auf eine Plattenosteosynthese gewechselt wurde. Auch hier zeigte sich eine insuffiziente anatomische Reposition, proximal der Fraktur waren die Schrauben ausschließlich monokortikal besetzt. Zudem war ein proximales Fragment nicht gefasst $(\mathbf{a}-\mathbf{g})$. Wir revidierten die Fraktur in unserer Klinik mittels anatomischer Reposition und winkelstabiler Plattenosteosynthese in Kombination mit einer interfragmentären Zugschraube. Das proximale Fragment wurde dabei mitgefasst und die Schrauben bikortikal aufsteigend subchondral besetzt $(\mathbf{h}, \mathrm{i})$. 
unterliegt [19]. Auf diese Weise ermöglichen die Fossae coronoidea wie auch radialis die Flexion und die Fossa olecrani die Extension im Ellenbogengelenk. Eine postoperative Malposition bzw. Überkorrektur reduziert das postoperative Bewegungsausmaß bzw. die Extensionskraft entsprechend. Ebenfalls muss in der chirurgischen Versorgung den am Epicondylus/Condylus humeri medialis und lateralis ansetzenden muskuloligamentären Stabilisatoren Rechnung getragen werden. Die am häufigsten genutzte Frakturklassifikation basiert auf der AOKlassifikation, die eine Separierung in extraartikulär (Typ A), partiell (Typ B) bzw. komplett intraartikulär (Typ C) vornimmt [19]. Die weitergehende Subtypisierung erteilt Aussagen über die Komplexität der Fraktur und liefert damit Hilfe für ein frakturspezifisches Vorgehen. Eine Sonderstellung nehmen die Abscherfrakturen in der Sagittalebene ein, deren Klassifikation über die Dubberley-Einteilung erfolgt [20]. Die nicht operative Versorgung ist nur Patienten mit sehr geringem Anspruch oder hohem perioperativem Risiko vorbehalten. Entsprechend der Literatur ist das Risiko eines inakzeptablen Ergebnisses um das 3-Fache erhöht und die Entstehung von Pseudarthrosen 6-mal häufiger beschrieben [21]. Für die meisten Patienten ist die operative Versorgung unbestritten, wobei der chirurgische Zugang von der Art der Fraktur abhängig ist. Uneinigkeit besteht weiterhin in der Anlage der Plattenosteosynthesen zueinander, wobei hier eine $90^{\circ}$ - vs. $180^{\circ}$-Konfiguration konkurrieren.

Bei nur limitierter intraartikulärer Beteiligung wie bei TypA-, -B1- und -B2-Frakturen erfolgt die Versorgung hauptsächlich über einen paratrizipitalen Zugang, der den Trizepssehnenansatz nicht kompromittiert. Besteht die Notwendigkeit für eine mehr artikularseitige Exposition, kann auf eine Olekranonosteotomie konvertiert werden. Alternative Zugänge bestehen mit dem Zugang nach Bryan und Morrey [22] oder Trizepssehnensplit mit Erweiterung des Zuganges entsprechend den Ausführungen von Campbell [23]. Bei Frakturen des medialen und lateralen Epicondylus humeri handelt es sich um Avulsionsfrakturen, die aufgrund des Muskelzuges reponiert und refixiert werden sollten. Über einen ulnaren oder radialen Zugang empfiehlt sich die Versorgung mittels Schraubenosteosynthese, wobei die Verwendung (sofern möglich) von 2 Schrauben die Rotationsstabilität deutlich erhöht. Partiell unikondyläre Frakturen können ebenfalls über einen radialen bzw. ulnaren Zugang mittels einseitiger Plattenosteosynthese versorgt werden. Die alleinige, häufig früher angewandte Schraubenosteosynthese ist von deutlich geringerer Stabilität und nicht mehr empfohlen. Die Versorgung von partiell artikulären frontalen Frakturen (Klassifikation nach Dubberley) erfolgt i.d.R. über eine Exposition von radial unter Beachtung des radialen kapsuloligamentären Bandapparates. Hierbei empfiehlt sich die Versorgung mit kopfversenkenden Minischrauben in a.-p. Richtung [24].
Typ-C-Frakturen erfordern i.d. R. eine Olekranonosteotomie mit der größtmöglichen Exposition der Gelenkfläche, die bei falscher Ausführung Komplikationspotenzial mit sich bringen kann [19]. Die Olekranonspitze wird V-förmig bis knapp über die Knorpelgrenze angesägt und die Osteotomie mit einem Meißel komplementiert. Als Hauptkomplikation ist hierbei der Implantatkonflikt zu erwähnen, der alternativ mit der Verwendung einer Zugschraube oder einer anatomisch vorgeformten winkelstabilen Doppelplattenosteosynthese bei kritischen Weichteilen umgangen werden kann [25]. Weitere mögliche Komplikationen bestehen in der Mal- bzw. Nonunion der Osteotomie bei fehlerhafter Einstellung. Möglichkeiten zur Reduktion der Komplikationsrate bestehen u. a. durch Bohrungen vor Osteotomieanlage und Verwendung von Repositionszangen bei Anlage der Osteosynthese. Der Goldstandard bei C-Frakturen ist die stufenfreie anatomische Gelenkrekonstruktion unter Verwendung von winkelstabilen Plattensystemen $(2,7 / 3,5 \mathrm{~mm})$ in $90^{\circ}$ oder $180^{\circ}$-Konfiguration. Im klassischen Vorgehen wird der Gelenkblock gestellt, temporär mit K-Drähten fixiert bzw. mit Zugschrauben stabilisiert. Die Reposition wird anschließend über die bikondyläre Plattenanlage gehalten. Mit zunehmender Komplexität der Frakturmorphologie wird die Rekonstruktion zur Herausforderung. In diesem Fall besteht das Vorgehen darin, das Hauptfragment zu definieren und mit Mini-Fragment-Schrauben die umliegenden Fragmente stabil zu refixieren. Bei großem Pfeilerfragment kann eine Reposition auch über das Pfeilerfragment erfolgen. In einigen Fällen ist eine komplette Rekonstruktion bei multifragmentärem Frakturcharakter nicht möglich, sodass ggf. eine Entfernung der Fragmente mit konsekutiver Verkürzung des distalen Humerus als Ausweg gewählt wird. Die erreichte Stabilität geht jedoch auf Kosten der Extensionskraft. Hughes et al. evaluierten, dass eine Verkürzung des distalen Humerus um $1 \mathrm{~cm}$ die Extensionskraft um $11 \%$, bei $3 \mathrm{~cm}$ um $21 \%$ bei $90^{\circ}$ Flexion reduziert [26]. Zusätzlich führt die Verkürzung der Trochlea zu einer Inkongruenz zwischen Trochlea und der Fossa olecrani mit konsekutiver Bewegungseinschränkung postoperativ. Idealerweise erfolgt die Plattenosteosynthese nach vollständiger Rekonstruktion und Refixation der Gelenkfläche. Im Hinblick auf die Plattenlänge ist, unabhängig von der Konfiguration, darauf zu achten, dass die Platten nicht auf derselben Höhe enden, um etwaige Stresskonzentrationen zu vermeiden $[24,27]$ ( $\triangleright$ Abb. 6).

Eine stabile osteosynthetische Versorgung von Low-PlaneFrakturen ist häufig aufgrund der geringen Knochensubstanz nicht adäquat möglich, sodass alternativ eine Ellenbogenprothese indiziert sein kann $[19,24]$. Trotz der lebenslangen Belastungseinschränkung von $5 \mathrm{~kg}$ ist das funktionelle Outcome besser vorhersehbar mit der endoprothetischen Versorgung. McKee et al. stellten in einem prospektiv randomisierten Studiendesign die Versorgung mittels Osteosynthese vs. Endoprothese für distale Humerusfrakturen Typ C gegenüber [28]. Basierend auf den 

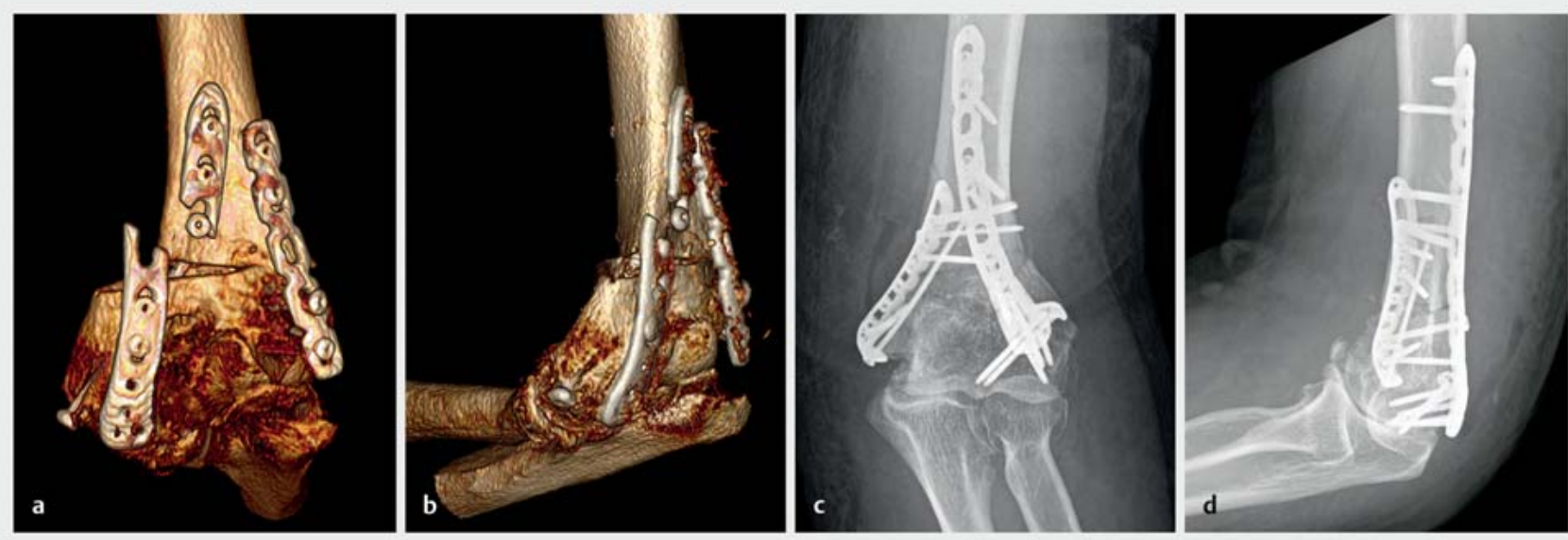

- Abb. 6 53-jähriger Patient mit posttraumatischer Arthrose nach Abriss des Epicondylus radialis humeri im Kindesalter. Auswärtige Versorgung mittels suprakondylärer Umstellungsosteotomie und Doppelplattenosteosynthese. Wenige Wochen postoperativ kam es zum Plattenbruch, am ehesten aufgrund der auf gleicher Höhe endenden Platten (a, b). Revision mittels anatomischer Reposition und winkelstabiler Doppelplattenosteosynthese, wobei beide Platten auf unterschiedlichen Höhen enden, um den biomechanischen Stress hier zu vermindern.

Studienergebnissen zeigte die Versorgung mittels Ellenbogenendoprothese mit einem Follow-up von 2 Jahren eine geringere Reoperationsrate mit einem besser vorhersagbaren Outcome [28]. Rajaee et al. wiesen auf eine signifikante Zunahme der endoprothetischen Versorgung für die letzten 10 Jahre für Patienten älter als 65 Jahre hin 29]. Aufgrund der Frakturmorphologie des distalen Humerus, welche die Bandansätze an den Epikondylen häufig inkludiert, führt die Wahl des Prothesendesign mehr zu Semiconstrained/Constrained Prothesen, da die Hemiprothesen eine aufwendige Rekonstruktion voraussetzen.

Die operative Versorgung der distalen Humerusfrakturen ist eine große Herausforderung unter dem Aspekt der komplexen Anatomie dieses Gelenkes. Henley et al. zeigten bei einer Patientenpopulation mit einem durchschnittlichen Alter von 32 Jahren eine $45 \%$ ige Komplikationsrate auf, obwohl 92\% der analysierten Patienten über ein exzellentes Outcome berichtet haben [30]. Bei der Hälfte der Komplikationen bestand eine Assoziation zur Olekranonosteotomie [30]. Huang et al. evaluierten die Resultate nach osteosynthetischer Versorgung bei Patienten älter als 65 Jahre. Ohne explizite Differenzierung der Frakturtypen konnte nur über exzellentes Outcome in $45 \%$ berichtet werden [31]. Unabhängig vom Patientenalter ist die anatomische, stabile Rekonstruktion der Schlüsselfaktor zur erfolgreichen Versorgung. Die Rate der in der Literatur beobachteten Pseudarthrosen wird mit $2-10 \%$ angegeben, wobei hauptsächlich die suprakondyläre Region involviert ist [32]. Die Revision besteht i.d. R. in einer Reosteosynthese, ggf. unter Verwendung von Autografts. Eine knöcherne Konsolidierung kann durchschnittlich 6 Monate nach der Revisionsoperation erreicht werden [33]. Häufig besteht additiv eine Ellenbogensteife, die im Rahmen der Revisionsoperation ebenfalls adressiert werden sollte, um die Belastungsspitzen auf das Implantat und die Pseudarthrosenregion zu reduzieren. Die Wiederholung der osteosynthetischen Versorgung in Kombination mit einem Weichteilrelease ist das ideale Revisionsvorgehen für junge, aktive Patienten. Ältere Patienten hingegen profitieren entsprechend den Ergebnissen von Ramsey et al. häufig von einer endoprothetischen Versorgung [34].

Neben klassischen Komplikationen wie Infektionen, heterotopen Ossifikationen, Kontrakturen und Ulnarisneuropathien ist ein Großteil der Revisionen auf die fehlgeschlagene Olekranonosteotomie zurückzuführen. Die optimale Gelenkexposition erfordert im Austausch ein zusätzliches osteosynthetisches Verfahren, das zu osteosyntheseassoziierten Komplikationen führen kann. Ein symptomatischer Implantatkonflikt wie auch die fehlende Konsolidierung zählen zu den häufigsten Komplikationen in diesem Bereich. In der Literatur werden über Nonunion-Raten von $11,9 \%$ berichtet, vorwiegend bei der Verwendung von Zuggurtungen [35]. Eine deutliche Reduktion der Nonunion-Rate wird bei plattenosteosynthetischer bzw. direkter Schraubenosteosynthese verzeichnet, jedoch ist häufiger eine Implantatentfernung notwendig [19].

\section{Ellenbogenluxationsfrakturen}

Komplexe Ellenbogenluxationsfrakturen mit Beteiligung des Radiuskopfes und/oder des Coronoids werden in ihrer Gesamtheit den Terrible-Triad-Verletzungen zugeschrieben und sind insgesamt mit einem wenig zufriedenstellenden Outcome vergesellschaftet. Die Radiuskopffraktu- 
ren sind bereits detailliert im vorhergehenden Kapitel besprochen worden. Aufgrund der Komplexität dieses Verletzungsmusters hier nur ein paar Bemerkungen zu den Coronoidfrakturen. Der Processus coronoideus ist ein wichtiger ulnohumeraler Gelenkstabilisator, dessen Funktion in der Vermeidung von posterioren ulnaren Subluxationen besteht sowie darin, posteromedialen und -lateralen Rotationskräften entgegenzuwirken. Da mehr als $60 \%$ des Coronoids durch die metaphysären Anteile der Ulna nicht supportiert werden, ist die Frakturwahrscheinlichkeit dieses ulnaren Anteils sehr hoch. Die weiterführende O’Driscoll-Klassifikation beschreibt neben Größe und Lokalisation der Fraktur zusätzlich die Beziehung zum MUCL, das am Tuberculum sublime inseriert. Typ-II-Frakturen involvieren die mediale Facette des Coronoids und sind damit relevant für die Varusstabilität des Ellenbogengelenkes. Aufgrund dessen ergibt sich die Rekonstruktionsnotwendigkeit von großen anteromedial lokalisierten Coronoidfragmenten, da diese signifikant zur Stabilität beitragen. Das Coronoid wird i.d.R. über einen medialen Zugang erreicht. Unter Beachtung der geografischen Nähe der neurovaskulären Strukturen kann entsprechend der Größe eine direkte oder indirekte osteosynthetische Versorgung vorgenommen werden. Im Rahmen der Komplettversorgung müssen sowohl der Radiuskopf als auch die kapsuloligamentären Strukturen wie LUCL und MUCL adressiert werden. Trotz eines gewissenhaften Managements ist das Komplikationsspektrum bei dieser Frakturkombinationsverletzung sehr hoch. Hauptkomplikationen sind hierbei Steife, rezidivierende Instabilität, posttraumatische Arthritis und Schmerz. Insbesondere die residuale Instabilität führt zeitig zu einer Degeneration des Ellenbogengelenkes. Um dies zu vermeiden, ist ein intraoperativ geplantes Vorgehen mit primärer Versorgung des Processus coronoideus, gefolgt von der Osteosynthese oder vom endoprothetischen Ersatz des Radiuskopfes sowie der Rekonstruktion des lateralen Bandapparates zielführend. Bei weiterhin bestehender Instabilität ist die Anlage eines Fixateur externe zu überdenken.

\section{Zusammenfassung}

Das Ellenbogengelenk unterliegt einer komplexen Anatomie, wobei die neurovaskulären Strukturen in enger Nachbarschaft zu den muskulären, ligamentären und knöchernen Schlüsseleinheiten stehen. Jegliches Trauma kann die anspruchsvolle Anatomie verändern und nachhaltig die Funktion des Ellenbogens beeinflussen. Die Ellenbogentraumatologie präsentiert sich mit einer weiten Diversität an Frakturen, wobei häufig auch eine Kombinationsverletzung knöcherner und kapsuloligamentärer Strukturen vorliegt, die bei Nichtadressierung neben dem Versagen der Osteosynthese auch zu weiteren Komplikationen wie persistierender Instabilität, Steife, Schmerzen und Ausbildung von heterotopen Ossifikationen führen kann. Vordergründig ist zumeist eine stabile, anato- mische Rekonstruktion unter Verwendung des entsprechenden chirurgischen Zuganges notwendig, um ein entsprechendes Osteosyntheseversagen im weiteren Sinne zu vermeiden.

\section{Interessenkonflikt}

Die Autorinnen/Autoren geben an, dass kein Interessenkonflikt besteht.

\section{Autorinnen/Autoren}
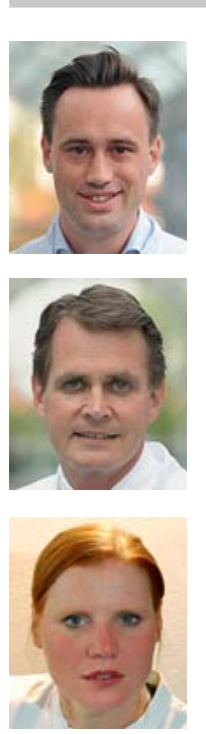

\section{Frank Graef}

Dr. med., Assistenzarzt, Charité-Universitätsmedizin Berlin, Centrum für Muskuloskeletale Chirurgie

\section{Ulrich Stöckle}

Univ.-Prof. Dr. med., Geschäftsführender Direktor, Charité-Universitätsmedizin Berlin, Centrum für Muskuloskeletale Chirurgie Foto: Wiebke Peitz

\section{Kathi Thiele}

Dr. med., Oberärztin, Charité-Universitätsmedizin Berlin, Centrum für Muskuloskeletale Chirurgie

\section{Korrespondenzadresse}

\author{
Dr. med. Frank Graef \\ Centrum für Muskuloskeletale Chirurgie \\ Charité Universitätsmedizin Berlin \\ Augustenburger Platz 1 \\ 13353 Berlin \\ Tel.: 030/450552012 \\ Fax: 030/450552901 \\ frank.graef@charite.de
}

Literatur

[1] Barco R, Antuña SA. Management of elbow trauma: anatomy and exposures. Hand Clin 2015; 31: 509-519

[2] Siebenlist S, Biberthaler P. Akute Kapsel-Band-Verletzungen des Ellenbogens. Trauma Berufskrankh 2015; 17: 132-139

[3] Armstrong AD, Ferreira LM, Dunning CE et al. The medial collateral ligament of the elbow is not isometric: an in vitro biomechanical study. Am J Sports Med 2004; 32: 85-90

[4] O'Driscoll SW, Jupiter JB, Cohen MS et al. Difficult elbow fractures: pearls and pitfalls. Instr Course Lect 2003; 52: 113-134

[5] Cohen MS, Hastings $\mathrm{H}$. Rotatory instability of the elbow. The anatomy and role of the lateral stabilizers. J Bone Joint Surg Am 1997; 79: 225-233

[6] O'Driscoll SW, Horii E, Morrey BF et al. Anatomy of the ulnar part of the lateral collateral ligament of the elbow. Clin Anat 1992; 5: 296-303 
[7] Rouleau DM, Faber K], Athwal GS. The proximal ulna dorsal angulation: A radiographic study. J Shoulder Elbow Surg 2010; 19: $26-30$

[8] Wong JC, Getz CL, Abboud JA. Adult Monteggia and olecranon fracture dislocations of the elbow. Hand Clin 2015; 31: 565580

[9] Goldfarb CA, Megan J, Patterson M et al. Elbow radiographic anatomy: measurement techniques and normative data. J Shoulder Elbow Surg 2012; 21: 1236-1246

[10] Tornetta P, Court-Brown C, Heckman J, McKee M, McQueen M, Ricci W. Rockwood and Green's Fractures in Adults. Philadelphia: Lippincott Williams \& Wilkins; 2014

[11] Caputo AE, Mazzocca AD, Santoro VM. The nonarticulating portion of the radial head: anatomic and clinical correlations for internal fixation. J Hand Surg Am 1998; 23: 1082-1090

[12] Mason ML. Some observations on fractures of the head of the radius with a review of one hundred cases. Br J Surg 1954; 42: 123-132

[13] Schmidt-Horlohé K, Siebenlist S, Stöckle U et al. [Fractures of the radial head and neck]. Z Orthop Unfall 2011; 149: e69e86

[14] Gierer P, Wichelhaus A, Rotter R. [Fractures of the olecranon]. Oper Orthop Traumatol 2017; 29: 107-114

[15] Powell AJ, Farhan-Alanie OM, Bryceland JK et al. The treatment of olecranon fractures in adults. Musculoskelet Surg 2017; 101: 1-9

[16] Bado JL. The Monteggia lesion. Clin Orthop Relat Res 1967; 50: 71-86

[17] Jupiter JB, Leibovic SJ, Ribbans W et al. The posterior Monteggia lesion. J Orthop Trauma 1991; 5: 395-402

[18] Korner ], Hoffmann A, Rudig L et al. [Monteggia injuries in adults: critical analysis of injury pattern, management, and results]. Unfallchirurg 2004; 107: 1026-1040

[19] Mighell MA, Stephens B, Stone GP et al. Distal humerus fractures. Hand Clin 2015; 31: 591-604

[20] Dubberley JH, Faber KJ, Macdermid JC et al. Outcome after open reduction and internal fixation of capitellar and trochlear fractures. J Bone Joint Surg Am 2006; 88: 46-54

[21] Nauth A, McKee MD, Ristevski B et al. Distal humeral fractures in adults. J Bone Joint Surg Am 2011; 93: 686-700

[22] Bryan RS, Morrey BF et al. Extensive posterior exposure of the elbow. A triceps-sparing approach. Clin Orthop Relat Res 1982; 166: 188-192

[23] Campbell WC. Incision for exposure of the elbow joint. Am J Surg 1932; 15:65-67

[24] Müller LP, Hollinger B, Burkhart K], Hrsg. Expertise Ellenbogen. Stuttgart: Thieme; 2016
[25] Ellwein A, Jensen G, Geerling J et al. New operative procedures on the elbow. Trauma Berufskrankh 2018; 20: 225-231

[26] Hughes RE, Schneeberger AG, An KN et al. Reduction of triceps muscle force after shortening of the distal humerus: a computational model. J Shoulder Elbow Surg 1997; 6: 444448

[27] Hackl M, Wegmann K, Taibah S et al. Peri-implant failure in dual plating of the distal humerus. A biomechanical analysis with regard to screw and plate positioning. Injury 2015; 46: 2142-2145

[28] McKee MD, Veillette CJH, Hall JA et al. A multicenter, prospective, randomized, controlled trial of open reduction-internal fixation versus total elbow arthroplasty for displaced intraarticular distal humeral fractures in elderly patients. J Shoulder Elbow Surg 2009; 18: 3-12

[29] Rajaee SS, Lin CA, Moon CN. Primary total elbow arthroplasty for distal humeral fractures in elderly patients: a nationwide analysis. J Shoulder Elbow Surg 2016; 25: 1854-1860

[30] Henley MB, Bone LB, Parker B. Operative management of intra-articular fractures of the distal humerus. J Orthop Trauma 1987; 1: 24-35

[31] Huang JI, Paczas M, Hoyen HA et al. Functional outcome after open reduction internal fixation of intra-articular fractures of the distal humerus in the elderly. J Orthop Trauma 2011; 25: 259-264

[32] Ring D, Gulotta L, Jupiter JB. Unstable nonunions of the distal part of the humerus. J Bone Joint Surg Am 2003; 85: 1040 1046

[33] Helfet DL, Kloen P, Anand N et al. Open reduction and internal fixation of delayed unions and nonunions of fractures of the distal part of the humerus. J Bone Joint Surg Am 2003; 85: 33-40

[34] Ramsey ML, Adams RA, Morrey BF. Instability of the elbow treated with semiconstrained total elbow arthroplasty. J Bone Joint Surg Am 1999; 81: 38-47

[35] Woods BI, Rosario BL, Siska PA et al. Determining the efficacy of screw and washer fixation as a method for securing olecranon osteotomies used in the surgical management of intraarticular distal humerus fractures. J Orthop Trauma 2015; 29: 44-49

Bibliografie

DOI https://doi.org/10.1055/a-1069-0605 online publiziert 23.03.2020 | OP-JOURNAL 2020; 36: 114122 @ Georg Thieme Verlag KG Stuttgart · New York ISSN 0178-1715 